\title{
High spin polarization at the interface between a Fe monolayer and $\operatorname{InAs(110)}$
}

\author{
L. Sacharow and M. Morgenstern \\ Institute of Applied Physics, Hamburg University, Jungiusstraße 11, D-20355 Hamburg, Germany \\ G. Bihlmayer and S. Blügel \\ Institut für Festkörperforschung, Forschungszentrum Jülich, D-52425 Jülich, Germany
}

(Received 30 October 2003; published 20 February 2004)

\begin{abstract}
The magnetic and electronic properties of a Fe monolayer on $\operatorname{InAs}(110)$ are calculated with an $a b$ initio method using the density-functional theory. We find that the relaxation of the $\operatorname{InAs}(110)$ surface is completely lifted and that the Fe atom is located in the center of the triangle formed of two As atoms and an adjacent In atom. The Fe bonding is largely determined by a molecularlike interaction of As and In orbitals with the Fe $d$ levels. In particular, the density of states at the Fermi level is dominated by a bond between minority $\mathrm{Fe} d_{x y}$, $d_{z y}$ levels and As $p_{x}$, In $s p$ levels. This leads to a large value of $80 \%$ spin polarization on the Fe side and $60 \%$ on the InAs side of the interface albeit of different orientation. Into the crystal the spin-polarization exhibits a damped oscillation.
\end{abstract}

DOI: 10.1103/PhysRevB.69.085317

PACS number(s): 73.21.Fg, 68.37.Ef, 73.20.At

For the fabrication of new electronic devices it is important to understand the interface properties between different materials. Of particular current interest is the interface between ferromagnets and semiconductors. For example, in order to realize the spin transistor proposed by Datta and Das, ${ }^{1}$ the interface between a ferromagnet and a semiconductor moved into the focus of research. From what is known today, the quality of the interface determines whether the injection of the spin-polarized electrons into the semiconductor is ballistic or diffusive. If it is diffusive, Schmidt et al. ${ }^{2}$ and Fert and Jaffrés ${ }^{3}$ have shown that the spin polarization of the current is negligibly small. Thus, a detailed understanding of the ferromagnet/semiconductor interface is crucial.

An experimental investigation of interfaces consisting of thick ferromagnetic films on semiconductors is rather difficult, so it might be appropriate to start with a lower thickness of the ferromagnet. Motivated by recent scanning-tunneling microscopy (STM) experiments on Fe atoms and chains on InAs(110), we calculate the atomic configuration, the magnetic properties, and the electronic structure of an interface consisting of a monolayer of $\mathrm{Fe}$ as a ferromagnetic material and $\operatorname{InAs}(110)$ as a semiconductor with large Rashba splitting. ${ }^{4,5}$ Understanding the properties of the monolayer paves also the path to use $\mathrm{Fe}$ for preparing nanostructures in order to move into the area of nanospintronics.

Although the relaxation properties at such interfaces are very important, they are often neglected in calculations considering spin injection properties. ${ }^{6,7}$ Here, we calculate the electronic and spin properties of a relaxed interface and find that molecularlike bonds between Fe and InAs atoms are formed. Surprisingly, this leads to a high spin polarization at both sides of the interface, in particular, at $E_{F}$.

First we calculated the structure of $\operatorname{InAs}(110)$, which exhibits the surface relaxation typical for III-V semiconductors, i.e., the As atom relaxes outwards and is 1.2 a.u. ( 1 a.u. $=0.529 \AA$ ) higher than the In atom. ${ }^{8}$ Then, we added one layer of Fe on the InAs(110) surface, and relaxed the atom positions to minimize the total energy. The resulting Fe position is in accordance with recent STM experiments. ${ }^{4} \mathrm{Fi}$ - nally, the spin-dependent band structure, the local density of states (LDOS) and the spatially integrated density of states (DOS) at the Fe/InAs interface were analyzed. This leads to the surprising conclusion that at $E_{F}$ the Fe spin-polarization is much higher for a monolayer Fe on $\operatorname{InAs}(110)(80 \%)$ than for bulk $\mathrm{Fe}(40 \%) .{ }^{9}$ Moreover, singletlike couplings of As $p_{x}$ and In $s p$ states to the $\mathrm{Fe} d_{x y}$ and $d_{z y}$ orbitals lead to a similar high spin polarization of different sign on the InAs side.

The calculations are performed using the densityfunctional theory. ${ }^{10}$ The exchange-correlation functional is formulated within the local spin-density approximation. ${ }^{11}$ The Kohn-Sham equations are solved applying the fullpotential linearized plane-wave method, ${ }^{12}$ as realized in the FLEUR-code. ${ }^{13}$ For simulating the InAs(110) surface we use a slab geometry with five layers of InAs embedded in infinite vacua on both sides of the slab. Tests with five and nine atomic layer slabs show that the energetically favorable structure is reproduced with sufficient accuracy using a five layer slab. Next, an $\mathrm{Fe}$ monolayer with one $\mathrm{Fe}$-atom per unit cell is placed on one side of the optimally relaxed InAs slab. For the optimization of the new configuration, the Femonolayer and the two adjacent layers of the InAs are allowed to relax.

The volume of the slab is partitioned into muffin-tin (MT) spheres and the interstitial region. To prevent an overlap of the MT-spheres and at the same time to take into account the extension of the states at the atoms we choose the radii of the MT's to be 2.2 a.u. for the Fe, 2.0 a.u. for the In and 1.8 a.u. for the As. The wave functions are expanded into augmented plane waves with a maximum $K$ vector of $K_{\max }$ $=3.9$ a.u. $^{-1}$, which amounts to 230 basis functions per atom. The basis function in the MT's are expanded into radial functions and spherical harmonics with angular momenta up to $l=8$. The Brillouin zone (BZ) integration was carried out using $121 k_{\|}$points in the irreducible wedge of the two-dimensional BZ.

The starting position for the relaxation of the $\mathrm{Fe}$ atom is deduced as follows: We compare measured STM images of 


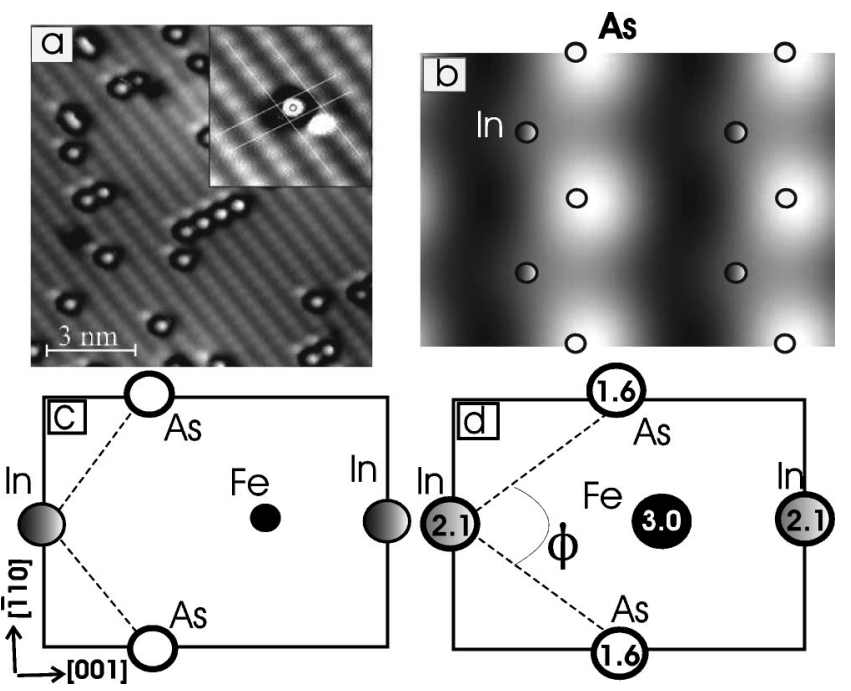

FIG. 1. (a) STM constant-current image of $\operatorname{InAs}(110)$ covered with $7.5 \% \mathrm{Fe}, U=50 \mathrm{mV}, I=200 \mathrm{pA}, T=8 \mathrm{~K}$ (Ref. 4); (b) calculated constant-current image of the relaxed InAs(110) surface, $U$ $=50 \mathrm{mV}$; the band bending of $300 \mathrm{meV}$ is taken into account (Ref. 15); (c) from (a) and (b) deduced position of the Fe atom in the $\operatorname{InAs}(110)$ unit cell; (d) calculated position of $\mathrm{Fe}$ atom in the InAs(110) unit cell after relaxation; The numbers on the atoms indicate the vertical relaxation in atomic units from the ideal bulk terminated position of the surface As. The angle $\phi$ is given in Table II.

InAs(110) covered with submonolayers of Fe with calculated STM images of clean InAs(110). ${ }^{14,8}$ Figure 1(a) shows the measured image. ${ }^{4}$ The atomic rows of one type of atoms are visible in the background. The bright spots surrounded by a black rim are the Fe atoms. The inset shows that the position of the Fe maxima is in between two atomic rows and slightly displaced towards one of them. Moreover, the Fe maxima are located exactly between two neighboring maxima inside the InAs rows. ${ }^{4}$ The calculated image of the clean $\operatorname{InAs}(110)$ surface at the same voltage is displayed in Fig. 1(b). For the calculation, the tip-induced band bending determined to be $300 \mathrm{meV}$ is taken into account. ${ }^{15}$ The marked atomic centers of the In and the As atoms reveal that the protrusions in the constant-current image correspond to the As atoms. This is opposite to the conventional knowledge that cations (In) are imaged at positive voltage on III-V materials, but has its origin in the high-energy position of the In dangling-bond state at $0.9 \mathrm{eV}$ above the conduction-band minimum and the fact that the surface As atoms are relaxed outwards. ${ }^{8,14}$ From comparison of Figs. 1(a) and 1(b) we deduce a lateral position of the Fe atom as displayed in Fig. 1(c). The optimization process of this atomic structure led to the structure shown in Fig. 1(d). We carefully checked that starting with other initial positions of the Fe atom leads either to the same final position or to final configurations with significantly higher energies.

Figure 2 shows a side view of the InAs film after relaxation. The lower half of the film shows the relaxation without the Fe monolayer, while the relaxed structure with $\mathrm{Fe}$ is shown in the upper half. There, in contrast to the film without Fe, In, and As are nearly at the same height. The In atom

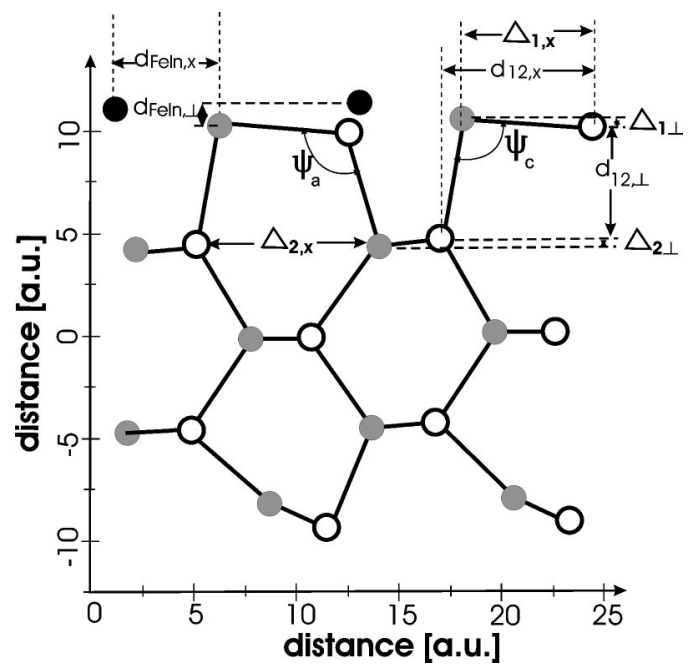

FIG. 2. Side view of calculated relaxation positions of InAs(110) surface with Fe monolayer. The lower half on the film shows the relaxation of the clean InAs(110) surface. Black circles mark the Fe positions, gray the In and white the As positions. Tables I and II give the corresponding values of distances, bond lengths, and angles.

is even slightly higher ( 0.5 a.u.) than the As atom. More importantly, the bond length between In and As in the uppermost layer as well as the bond length between the uppermost and the next layer of InAs are increased with respect to the bulk values and with respect to the relaxed InAs film without Fe. Tables I and II give the corresponding values of distances and bond lengths for the clean relaxed InAs(110) surface and the relaxed InAs(110) surface covered with one Fe atom per unit cell. The results for the clean surface are in excellent agreement with other theoretical work on III-V semiconductors. ${ }^{8}$ A strongly increased In-As bond length in the $\mathrm{Fe} / \mathrm{InAs}(110)$ case shows that In-As-bonds are weakened in favor of Fe-In and Fe-As bonds. ${ }^{16}$ Moreover, Fe is located nearly equidistant to the adjacent two As and the In atom as visible in Fig. 1(d). This indicates that bond formation to both surface species takes place.

TABLE I. Distances between the atoms in the clean relaxed InAs(110) surface and the InAs(110) surface covered with one Featom per unit cell according to the definitions in Fig. 2. The distances are expressed in units of the respective InAs bulk distances given in the second column.

\begin{tabular}{ccccc}
\hline \hline Distance & Unit & InAs bulk & InAs(110) & Fe/InAs(110) \\
\hline$a$ & {$[a \cdot u]$.} & 11.437 & & \\
$d_{\text {FeIn, }}$ & {$\left[\frac{1}{2} a / \sqrt{2}\right]$} & & & 0.079 \\
$d_{\text {FeIn, } x}$ & {$[a]$} & & & 0.429 \\
$\Delta_{1, \perp}$ & {$[a / \sqrt{2}]$} & 0.000 & -0.164 & 0.056 \\
$\Delta_{1, x}$ & {$\left[\frac{3}{4} a\right]$} & 1.000 & 1.033 & 0.632 \\
$d_{12, \perp}$ & {$\left[\frac{1}{2} a / \sqrt{2}\right]$} & 1.000 & 1.118 & 1.241 \\
$d_{12, x}$ & {$\left[\frac{1}{2} a\right]$} & 1.000 & 1.080 & 1.266 \\
$\Delta_{2, \perp}$ & {$[a / \sqrt{2}]$} & 0.000 & -0.018 & 0.043 \\
$\Delta_{2, x}$ & {$\left[\frac{3}{4} a\right]$} & 1.000 & 0.999 & 0.996 \\
\hline \hline
\end{tabular}


TABLE II. Comparison of relaxation angles and bond length changes between the InAs(110) surface and Fe/InAs(110) surface. The angles $\psi_{a}$ and $\psi_{c}$ are defined in Fig. 2 and $\phi$ in Fig. 1(d). $\delta c_{i} a_{j}$ are the changes of the bond lengths between the cation of the $i$ th layer and the anion of the $j$ th layer relative to the cation-anion InAs bulk bond length.

\begin{tabular}{lcccccc}
\hline \hline \multirow{1}{*}{ System } & $\begin{array}{c}\phi \\
{\left[{ }^{\circ}\right]}\end{array}$ & $\begin{array}{c}\psi_{a} \\
{\left[{ }^{\circ}\right]}\end{array}$ & $\begin{array}{c}\psi_{c} \\
{\left[{ }^{\circ}\right]}\end{array}$ & $\begin{array}{c}\delta c_{1} a_{1} \\
{[\%]}\end{array}$ & $\begin{array}{c}\delta c_{2} a_{1} \\
{[\%]}\end{array}$ & $\begin{array}{c}\delta c_{1} a_{2} \\
{[\%]}\end{array}$ \\
\hline bulk & 109.5 & 109.5 & 109.5 & 0.0 & 0.0 & 0.0 \\
InAs(110) & 110.9 & 90.1 & 123.7 & -0.7 & -0.1 & 0.5 \\
Fe/InAs(110) & 67.7 & 105.1 & 96.7 & 47.9 & 11.43 & 13.1 \\
\hline \hline
\end{tabular}

Next, we analyze the electronic properties for the optimized atomic configuration. Figure 3 shows the spindependent LDOS inside the MT's for the different atoms at the Fe/InAs interface. The LDOS of Fe exhibits one narrow peak in each spin channel, which is caused by the narrow two-dimensional $d$ bands of different spin. The majority peak is found around $-2.4 \mathrm{eV}$ and the minority one around $0.1 \mathrm{eV}$ giving an exchange splitting of about $2.5 \mathrm{eV}$. The magnetic Fe moment amounts to $2.6 \mu_{B}$ in the Fe MT sphere.

In contrast, the magnetic moments of the spheres of the In and As atoms are negligibly small, although both atoms show a strongly energy-dependent spin polarization, which is defined as the difference of the local spin $\uparrow$ and spin $\downarrow$ densities, divided by the spin-integrated LDOS, i.e., $(n \uparrow$ $-n \downarrow) /(n \uparrow+n \downarrow)$. This can be clearly seen in Fig. 3(c), where the spin polarization of the different atoms is displayed. Both atoms show a polarization up to $75 \%$, however, partly at different energies. Note that a rather high spin polarization is found around $E_{F}$. Interestingly, the peaks in Figs. 3(a) and 3(b) can be largely identified with particular atomic orbitals. To assign the peaks we analyzed the spatial distribution of the spin-polarized LDOS. The results with emphasis on the peaks close to $E_{F}$ are given in Fig. 4. Different cuts through the LDOS are shown and labeled according to the atoms through which the cut is taken (Fe, In, As) and according to the cutting plane $(x, y, z)$. Here, $x, y$, and $z$ are associated to the [001], [110], and [110] direction, respectively.

The different minority $d$ orbitals of the $\mathrm{Fe}$ atom are clearly recognizable in Figs. 4(a)-4(e). As indicated, we find $d_{x^{2}-y^{2}}$ symmetry around $-0.4 \mathrm{eV}$, overlapping $d_{z x}, d_{z y}$, and $d_{x y}$ states around $E_{F}$, and a $d_{z^{2}}$ orbital at $0.2 \mathrm{eV}$. The remaining Fe minority peak at $1 \mathrm{eV}$ contains only $d_{z y}$ and $d_{x y}$ orbitals (not shown). Figures 3(a) and 3(b) show two peaks in the As LDOS around $E_{F}$ and at $1 \mathrm{eV}$. One of them is found in the As majority channel $\left(E_{F}\right)$ and the other in the As minority channel $(1 \mathrm{eV})$.

Figures 4(f) and 4(i) demonstrate that the As peaks at $E_{F}$ and $1 \mathrm{eV}$ are the $p_{x}$ orbitals of different spin. We checked that the $p_{x}$ symmetry of the As states does not change within these two peaks. Therefore, we conclude that these two As peaks as well as the corresponding Fe peaks represent a singlet and a triplet of molecularlike states consisting of As $p_{x}$ and Fe $d_{x y} / d_{z y}$ orbitals. The selection of these $d$ levels is
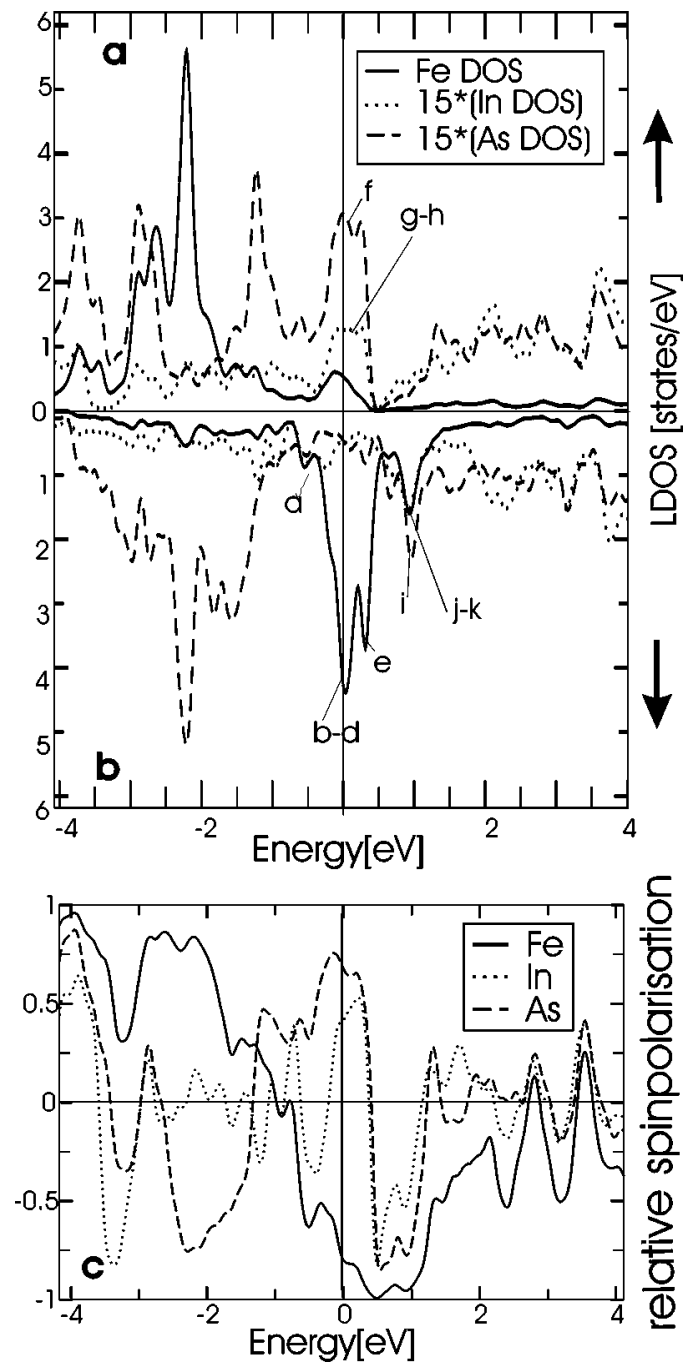

FIG. 3. (a), (b) spin-resolved LDOS inside the MT spheres of Fe (solid line), surface As (dashed line), and surface In (dotted line); LDOS of As and In are multiplied by a factor of 15 to display them on the same scale as the Fe LDOS; (a) majority spin ( $\uparrow$ ); (b) minority spin $(\downarrow)$; (c) energy dependence of the resulting relative spin polarization $((n \uparrow-n \downarrow) /(n \uparrow+n \downarrow))$; the labels in (a) and (b) refer to the LDOS images in Fig. 4.

reasonable, since only they exhibit a large overlap with the As atoms [see Fig. 4(b) and 4(d)].

The distinction of singletlike and tripletlike states can also be seen in the plot of the spin polarization shown in Fig. 3(c). The Fe spin polarization mainly exhibits two distinct regions of different sign with the change of sign around $-0.8 \mathrm{eV}$. This coincides with the energy regions of majority and minority $d$ bands. Importantly, in both energy regions basically two different spin polarizations of As are found. They correspond to the singlet and the triplet region, respectively. Consecutively, triplet $(\mathrm{Fe} \uparrow)$, singlet $(\mathrm{Fe} \uparrow)$, singlet (Fe $\downarrow$ ) and triplet $(\mathrm{Fe} \downarrow)$ regions are found. The detailed behavior within the majority (Fe $\uparrow$ ) band is more complex than in the $(\mathrm{Fe} \downarrow)$ band, but again can be traced back to a singlet-triplet splitting, now between bonds containing As $p_{x}, p_{y}$, and $p_{z}$ orbitals and Fe $d$ levels. Finally, the spin-up As peak around $-1.2 \mathrm{eV}$ is identified as a $p_{z}$ orbital of As 


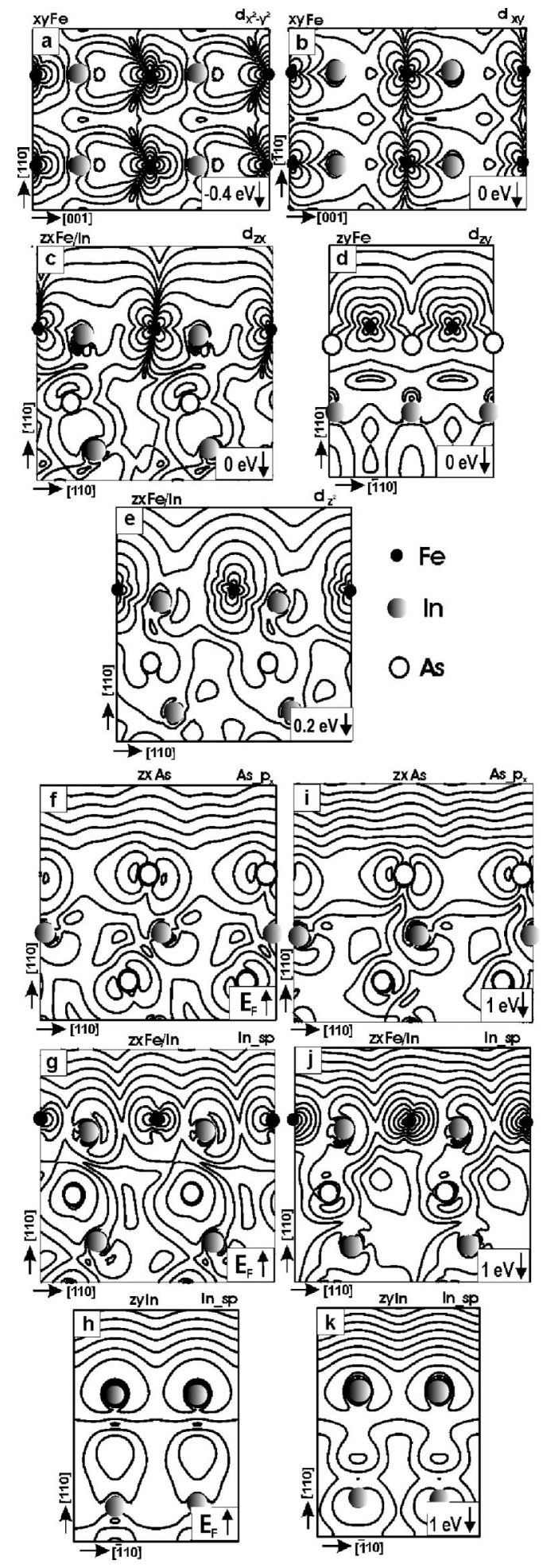

FIG. 4. Planar cuts through the $\log (\mathrm{LDOS})$ taken at different energies and spin orientations as indicated; labels on the left above the images mark the orientation of the plane $(x, y, z)$ and the atoms through which the plane is cut $(\mathrm{Fe}, \mathrm{In}, \mathrm{As})$, labels on the right indicate the identified orbital; black circles mark the Fe positions, gray the In-positions and white filled circles the As positions; (a)(e) minority LDOS taken $50 \mathrm{meV}$ around the indicated energies; (f) -(h) LDOS of the majority spin integrated from $-0.3 \mathrm{eV}$ to $0.3 \mathrm{eV}$; (i) $-(\mathrm{k})$ LDOS of the minority spin integrated from $0.8 \mathrm{eV}$ to $1.1 \mathrm{eV}$.

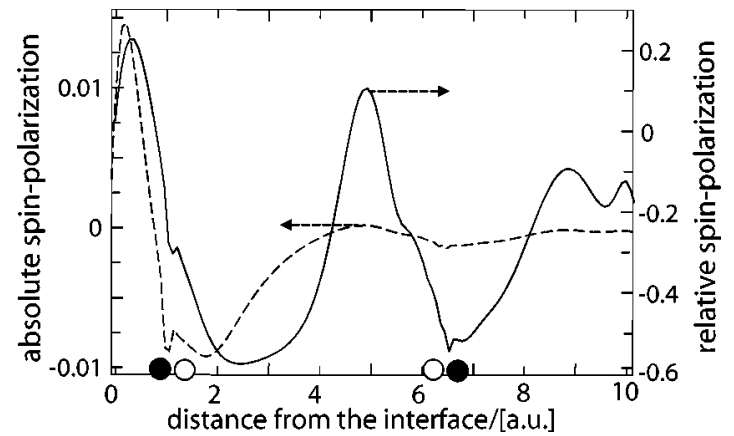

FIG. 5. Distance dependence of spin-polarization at $E_{F}$, integrated over both the muffin tin and the interstitial region; the circles at the bottom mark the positions of In- (black) and As-atoms (white). The dashed curve represents the absolute spin-polarization $(n \downarrow-n \uparrow)$ and the solid curve the relative spin polarization ( $n \downarrow$ $-n \uparrow) /(n \downarrow+n \uparrow)$. Note that the curves display the LDOS of only two calculated states (Kohn-Sham orbitals) near $E_{F}$.

not interacting with the Fe. We conclude that As atoms exhibit a nearly rectangular configuration of $p_{x}, p_{y}$, and $p_{z}$ orbitals, from which at least the $p_{x}$ orbitals are largely spin polarized due to a molecularlike interaction with the spinpolarized Fe $d$ levels.

Since we also observe spin polarization at the In atoms [Fig. 3(c)], there must also be an interaction of the Fe with the In levels. Indeed, Figs. 3(a) and 3(b) show coincidences of In and Fe peaks. In particular, tripletlike In couplings to the Fe peaks at $1 \mathrm{eV}$ and $-3.5 \mathrm{eV}$ and a singletlike coupling close to $E_{F}$ are found. The In peaks at $E_{F}$ and $1 \mathrm{eV}$ are again a singlet-triplet pair involving an In $s p$ level visible in the LDOS images of Figs. 4(g)-4(k). Importantly, we identify the interface LDOS at $E_{F}$ as due to a singletlike interaction of Fe $d$ levels with As $p_{x}$ and In $s p$ levels.

Since the spin polarization at $E_{F}$ is decisive for spintronic devices, we discuss it more in detail. As visible in Fig. 3(c), it is $80 \%$ within the Fe MT's and also large, but of opposite sign in the In and As MT's at the interface. Figure 5 shows the relative $(n \downarrow-n \uparrow) /(n \downarrow+n \uparrow)$ and the absolute spin polarization $(n \downarrow-n \uparrow)$ at $E_{F}$ in the InAs layer as a function of distance from the interface. Here, the MT's as well as the interstitial region is considered. The spin polarization close to the interface is opposite to the Fe spin-polarization highlighting the singletlike bond at this energy. More importantly, the relative spin-polarization exhibits a damped oscillation with atomic periodicity having values as large as $60 \%$.

In summary, we calculated the electronic and spin properties of a Fe monolayer on InAs(110) using the local-density approximation. We find that Fe takes a position equidistant to two As atoms and an In atom and moves all surface atoms outwards with respect to the clean InAs(110) surface. Molecularlike bonds exhibiting singlet-triplet splitting are identified. In particular, a singlet between As $p_{x}$, In $s p$, and Fe $d$ levels is found at $E_{F}$ and its triplet counterpart, again consisting of As $p_{x}$, In $s p$, and Fe $d$ levels, is found at $0.9 \mathrm{eV}$. The molecular bonding leads to a large spin polarization in the Fe layer as well as in the InAs layer. At $E_{F}$, the spin polarization in the InAs is found to oscillate with distance from the interface having values as large as $60 \%$. These re- 
sults clearly show that details of electronic and magnetic properties of ferromagnet/semiconductor interfaces can be significantly different from the pure materials. Further studies of spin injection properties resulting from such a high spin polarization would be quite interesting.
We thank R. Wiesendanger and S. Heinze for helpful discussions. Financial support from SFB Grant No. 508-B4, Graduiertenkolleg "Physik nanostrukturierter Festkörper" and project Wi 1277/15-2 of the Deutsche Forschungsgemeinschaft is gratefully acknowledged.
${ }^{1}$ S. Datta and B. Das, Appl. Phys. Lett. 56, 665 (1990).

${ }^{2}$ G. Schmidt, D. Ferrand, L.W. Molenkamp, A.T. Flip, and B.J. van Wees, Phys. Rev. B 62, R4790 (2000).

${ }^{3}$ A. Fert and H. Jaffrés, Phys. Rev. B 64, 184420 (2001).

${ }^{4}$ M. Morgenstern, M. Getzlaff, D. Haude, and R. Wiesendanger, Phys. Rev. B 61, 13805 (2000).

${ }^{5}$ T. Matsuyama, R. Kürsten, C. Meissner, and U. Merkt, Phys. Rev. B 61, 15588 (2000).

${ }^{6}$ P. Mavropoulos, O. Wunnicke, and P.H. Dederichs, Phys. Rev. B 66, 024416 (2002).

${ }^{7}$ M. Zwierzycki, K. Xia, P.J. Kelly, G.E.W. Bauer, and I. Turek, arXiv.org/archive/cond-mat (unpublished).

${ }^{8}$ B. Engels, P. Richard, K. Schröder, S. Blügel, P. Ebert, and K. Urban, Phys. Rev. B 58, 7799 (1998).
${ }^{9}$ B. Sinković and E. Shekel, Phys. Rev. B 52, 8696 (1995).

${ }^{10}$ P. Hohenberg and W. Kohn, Phys. Rev. 136, 864 (1964).

${ }^{11}$ S.H. Vosko, L. Wilk, and M. Nusair, Can. J. Phys. 58 (8), 1200 (1980).

${ }^{12}$ E. Wimmer, H. Krakauer, M. Weinert, and A. Freeman, Phys. Rev. B 24, 864 (1981).

${ }^{13} \mathrm{http}: / / w w w . f l a p w . d e$

${ }^{14}$ J. Klijn, L. Sacharow, C. Meyer, S. Blügel, M. Morgenstern, and R. Wiesendanger, Phys. Rev. B 68, 205327 (2003).

${ }^{15}$ R. Dombrowski, C. Steinebach, C. Wittneven, M. Morgenstern, and R. Wiesendanger, Phys. Rev. B 59, 8043 (1999).

${ }^{16}$ S.C. Erwin, S.-H. Lee, and M. Scheffler, Phys. Rev. B 65, 205422 (2002). 